\title{
Outcomes using the llizarov external mini-fixator for Monteggia fractures in children
}

\author{
K. Li ${ }^{1}$, S. Rong ${ }^{1}$, C. Zheng ${ }^{1}$, Y. Teng ${ }^{2}$, H. Li ${ }^{1}$, L. Liu ${ }^{1}$, K. Zhen ${ }^{1}$, X. Shen ${ }^{1}$, J. Feng ${ }^{1}$, F. Li ${ }^{1}$ \\ 'The Third Hospital of Shijiazhuang City, Shijiazhuang, China \\ ${ }^{2}$ The General Hospital of Xinjiang Military Region, Urumqi, China
}

\begin{abstract}
Objective To evaluate the use of Ilizarov external mini-fixation in the treatment of Monteggia fractures (dislocation of the radial head with an associated fracture of the proximal ulna) in children. Methods Children with proximal ulnar fracture were included and underwent fracture reduction surgery with Ilizarov external mini-fixators, followed by immobilization of the supinated forearm with plaster. The reduction was evaluated intra-operatively using arthrography. Mackay criteria were used to evaluate clinical outcomes at follow-up. Results A total of 15 children were included in the study. Mackay efficacy was $100 \%$, indicating excellent outcomes using the Ilizarov external mini-fixator. Conclusion Use of the Ilizarov external mini-fixator is particularly suitable in the treatment of children with comminuted and compression fractures of proximal ulna. It is easy to operate, low invasive and is worthy of promotion.
\end{abstract}

Keywords: children, ulna, Monteggia fractures, Ilizarov mini-fixator, arthrography, plaster immobilization

\section{INTRODUCTION}

Studies on the pathology, classification, diagnosis, and treatment of fractures of the proximal ulna, Monteggia fractures, in children have been published for more than a century [1]. However, this type of fracture in children still poses challenges for orthopedic surgeons. The treatment principles for Monteggia fractures repair in children is to correct ulnar deformity, maintain the length of the ulna, restore the humeroradial joint, and ensure the stability of the fracture. A suitable surgical method must be chosen on the basis of these treatment principles.

The goal of this study was to evaluate whether Ilizarov external mini-fixation is suitable for the treatment of Monteggia fractures in children.

\section{METHODS}

\section{General information}

Children with proximal ulnar fractures who were diagnosed and treated at our department from 2018 to 2020 were eligible for the study. Children who were included ( $\mathrm{n}=15 ; 6$ left, 9 right side; 10 males, 5 females; age range 3-9 years, average 6.5 years) underwent surgical treatment for Monteggia fractures (Bado classification types: 7 type I, 0 type II, 8 type III, 0 type IV; types of ulnar fracture: 4 green branch, 6 transverse and short oblique fractures, 5 comminuted fractures, 2 combined with radial nerve contusion). All had intraoperative elbow arthrography and were followed-up to evaluate the therapeutic outcome.

Surgical methods

(1) General anesthesia was used for all patients. (2) Elbow arthrography was performed. An allergy test was administered to verify that the patient was not allergic to the contrast medium (iohexol); iohexol $(1-1.5 \mathrm{~mL}$ ) was injected into the elbow joint cavity to facilitate even dispersion of the contrast medium. C-arm fluoroscopy was used to view the alignment and position of the bones at the elbow during reduction of the humeroradial joint. (3) To perform fixation with the Ilizarov external minifixator, a 2-cm longitudinal incision was made along the dorsal ulna, beginning proximal to and with the fractured end at its center. The ulna was manipulated and the bone alignment and length was restored. Two Kirschner wires were inserted longitudinally through the olecranon and into the distal and proximal ends of the fracture to establish support points. The nail clips were installed, and a threaded rod was connected. If the ulnar bone shape was restored, but arthrography showed poor reduction of the humeroradial joint (such as soft tissue embedded within the humeroradial joint), open reduction was performed. (4) After surgery, the forearm was immobilized with plaster in a supinated position for 4-6 weeks.

\section{Evaluation}

Postoperative function was evaluated using Mackay criteria[1]: excellent, if there was no dislocation of the humeroradial joint, recovery of neurological symptoms, a normal range of elbow flexion-extension, and a normal range of forearm rotation; good, if there was no dislocation of the humeroradial joint, elbow extension-flexion was limited by $<20^{\circ}$, and forearm rotation was limited by approximately $10^{\circ}$; and poor, if the humeroradial joint was dislocated or subluxated, elbow extension-flexion was limited by $>50^{\circ}$, and forearm rotation was limited by $>50^{\circ}$. 


\section{RESULTS}

Children included in the study were followed for 6-18 months; 12 children had excellent outcomes $(80 \%), 3$ children had good outcomes (20\%), and none had poor outcomes $(0 \%)$. Two children who had preoperative radial nerve injury recovered after strengthening functional exercises and oral mecobalamin treatment. No infections, ischemic muscle contracture, myositis ossificans, fracture nonunion, or delayed fracture union occurred in any of the children in the study. A typical case is shown in Figure 1.
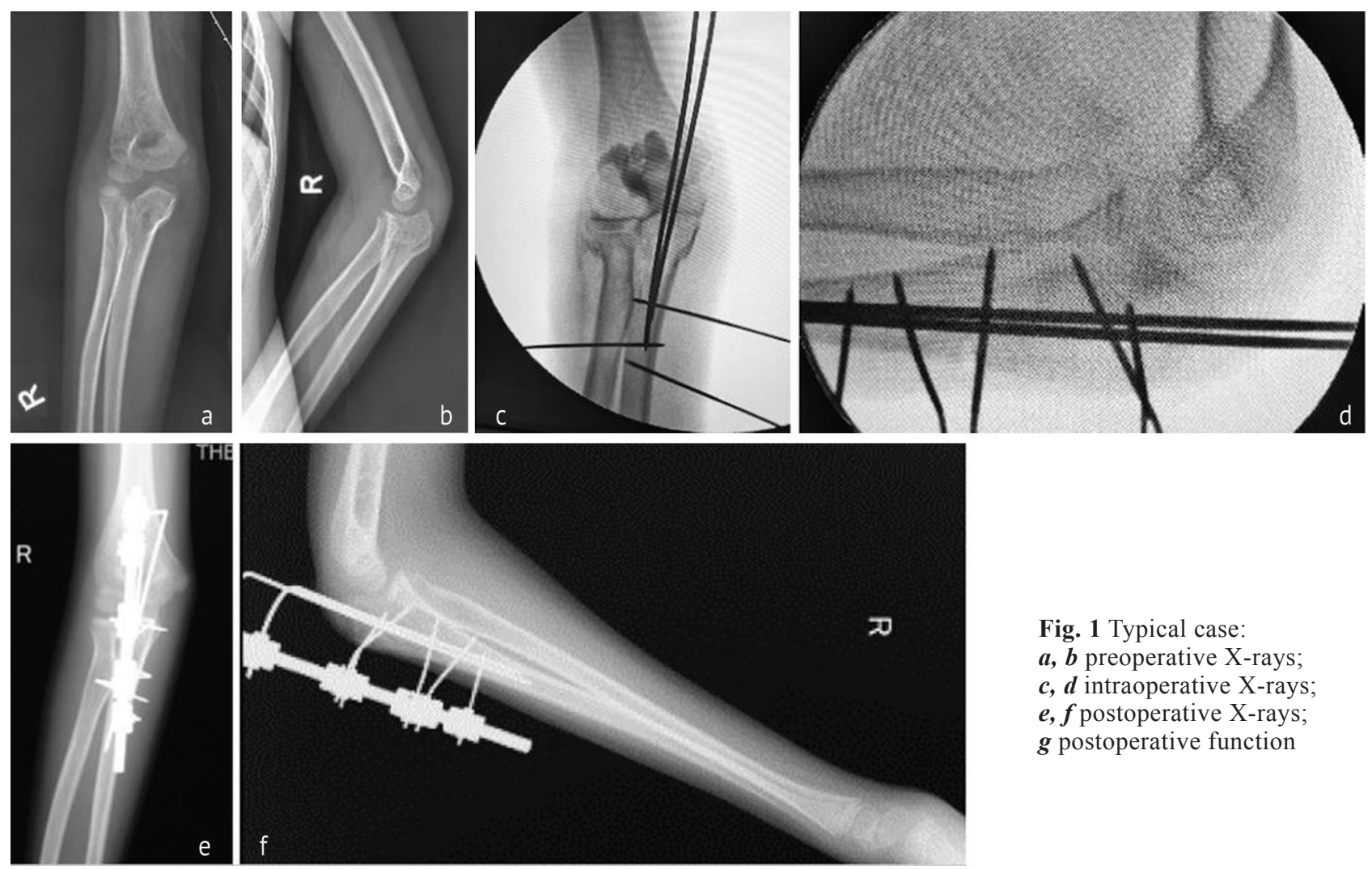

Fig. 1 Typical case:

$\boldsymbol{a}, \boldsymbol{b}$ preoperative X-rays;

$\boldsymbol{c}, \boldsymbol{d}$ intraoperative X-rays;

$\boldsymbol{e}, \boldsymbol{f}$ postoperative $\mathrm{X}$-rays;

$g$ postoperative function
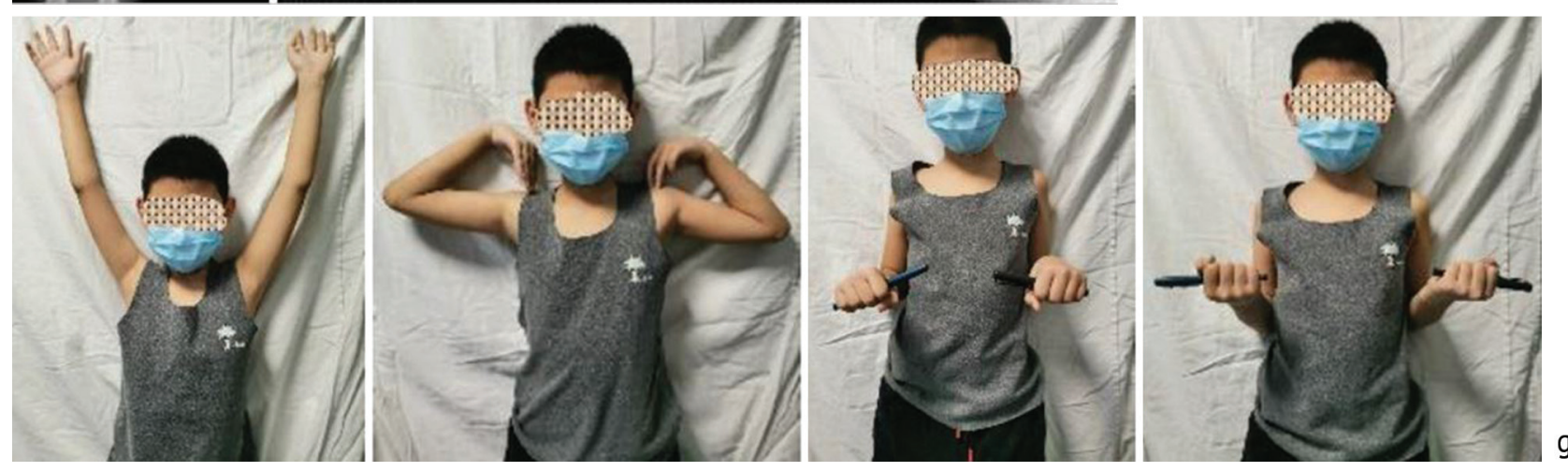

\section{DISCUSSION}

Literature on Monteggia fractures in children establishes thorough knowledge and understanding of the treatment principles for Monteggia fractures repair in children which are to correct ulnar deformities, maintain the length of the ulna, reduce the fracture, and ensure humeroradial joint stability. Elbow arthrography can be used to clearly show the cartilage structures of the distal humerus, proximal ulna, and proximal radius and the relative alignment of the humeroradial joint to correct mild subluxation and improve the diagnosis of potential complications when reducing the fracture and to choose an appropriate fixation method based on the type of ulnar fracture. For curved and incomplete ulnar fractures, a closed reduction may be performed, and plaster fixation can be used. For transverse and short oblique ulnar fractures, elastic intramedullary nails can be used for fixation, and long oblique and comminuted ulnar fractures may be fixed with steel plate on screws [2].

Certain challenges exist in treating Monteggia fractures in children $[3,4]$. The proximal ulna components, the olecranon, trochlear notch, and coronoid process are composed of cancellous bone; thus fractures are prone to local fragmentation, angulation, and compression. Often, manual reduction is difficult, and open reduction is required. In older adolescents, bone development is in its later stages; therefore, ossification of the proximal 
ulna is nearly complete, and the local space is large to fix. Consequently, the choice of fixation is a plate with screws or elastic intramedullary nail, and is based solely on the type of ulnar fracture. However, in young children, local bone space at the proximal end of the ulna is limited, and plate fixation requires the removal of bone from both ends of the fracture, which causes additional trauma. In addition, poor placement of the plate and screws can easily damage the olecranon epiphysis and articular surface (trochlear notch), causing subsequent injury and deformity. During elastic intramedullary nail fixation surgery, creating the intramedullary opening can also damage the epiphysis and articular surface, and if the proximal moment arm is too short, stability will be poor. In adults, use of Kirschner-wire tension-band fixation for olecranon fractures is standard [5], and it can also be used to treat Monteggia fractures in children but compresses the fracture longitudinally and provides no support. Therefore, it can only be used for transverse, non-comminuted, non-compression proximal ulnar fractures; otherwise it will cause compression of the fracture ends, will reduce ulnar length, and may increase the risk of humeroradial dislocation. Thus, the choice of internal fixation for younger children should be based not only on the type of ulnar fracture, but also on considerations such as the amount of bone space at the proximal end of the fracture.

The Ilizarov external mini-fixator has been currently used mainly for correction of metacarpal and phalangeal fractures and hand deformities [6-9]. In China, its current use is rare, and there is a lack of relevant literature. Director Li Kewei of our department trained at the Ilizarov Center in Kurgan, Russia in 2018. After returning to China, the Ilizarov external mini-fixator started to be widely used in the correction of metacarpal and phalangeal fractures and hand deformities in children.

In our study, good clinical outcomes were achieved in children with Monteggia fractures with the Ilizarov mini-external fixator, which precluded excessive removal of the soft tissue surrounding the fracture and the risk of damage to the epiphysis with screw. It provided stable fixation, including strong support for comminuted and compression proximal ulnar fractures. Only a 2-cm incision is required; therefore, this surgical method causes minimal trauma.

\section{CONCLUSION}

The Ilizarov external mini-fixator may be used for Monteggia fractures in children in accordance with established treatment principles. It is an appropriate

surgical method based on the type of the ulnar fracture. The Ilizarov external mini-fixator can provide us with a new treatment idea.

Funded Projects Shijiazhuang City Science and Technology Research and Development Guidance Plan Project (No. 171462323); National Natural Science Foundation of China (No. 31360229, 51165044); Xinjiang Uygur Autonomous Region Regional Collaborative Innovation Special Project (No.2019E0277).

\section{REFERENCES}

1. Johnson N.P., Silberman M. Monteggia Fractures. Treasure Island (FL), StatPearls Publishing, 2021. Available from: https://www.ncbi.nlm.nih.gov/books/ NBK470575/

2. Flynn J.M., Skaggs D.L., Waters P.M. Rockwood and Wilkins' fractures in children. Eighth edition. Ed. by J.M. Flynn, D.L. Skaggs, P.M. Waters. Wolters Kluwer Health, 2014.

3. Korobeinikov A., Popkov D. Use of external fixation for juxta-articular fractures in children. Injury, 2019, vol. 50, no. Suppl. 1, pp. S87-S94. DOI: 10.1016/j. injury.2019.03.043

4. Gallone G., Trisolino G., Stilli S., Di Gennaro G.L. Complications during the treatment of missed Monteggia fractures with unilateral external fixation: a report on 20 patients in a 10-year period in a tertiary referral center. J. Pediatr. Orthop. B, 2019, vol. 28, no. 3, pp. 256-266. DOI: 10.1097/BPB.0000000000000592

5. Chalidis B.E., Sachinis N.C., Samoladas E.P., Dimitriou C.G., Pournaras J.D. Is tension band wiring technique the "gold standard" for the treatment of olecranon fractures? A long term functional outcome study. J. Orthop. Surg. Res., 2008, vol. 3, pp. 9. DOI: 10.1186/1749-799X-3-9

6. Kanchanathepsak T., Gotani H., Hamada Y., Tanaka Y., Sasaki K., Yagi H., Kubota Y., Tawonsawatruk T. The effectiveness of distraction lengthening in traumatic hand amputation with Ilizarov mini fixator. Injury, 2020, vol. 51, no. 12, pp. 2966-2969. DOI: 10.1016/j.injury.2020.02.080

7. Tomori Y., Nanno M., Kentrao S., Majima T. Minimally invasive corrective osteotomy with the Ilizarov mini-fixator for malunited fractures of the phalanges: a technical note. J. Nippon Med. Sch., 2020. DOI: 10.1272/jnms.JNMS.2021_88-314

8. Danilkin M.Y. Phalangeal Lengthening Techniques for Brachydactily and Posttraumatic Digital Stumps with the Use of a Modified External Mini-Fixator. Tech. Hand Up. Extrem. Surg., 2016, vol. 20, no. 2, pp. 61-66. DOI: 10.1097/BTH.0000000000000116

9. Zolotov A.S. Open Infected Seymour Fracture. J. Hand Surg. Asian Pac. Vol., 2019, vol. 24, no. 4, pp. 488-490. DOI: 10.1142/S2424835519720196

Received: 31.03 .2021

Information about the authors:

1. Kewei Li,

The Third Hospital of Shijiazhuang City, Shijiazhuang, China

2. Shuai Rong,

3. Chong Zheng,

The Third Hospital of Shijiazhuang City, Shijiazhuang, China

4. Yong Teng,

The General Hospital of Xinjiang Military Region, Urumqi, China

5. Hao Li,

The Third Hospital of Shijiazhuang City, Shijiazhuang, China

6. Liantao Liu,

The Third Hospital of Shijiazhuang City, Shijiazhuang, China
7. Kepei Zhen,

The Third Hospital of Shijiazhuang City, Shijiazhuang, China

8. Xiaoyu Shen,

The Third Hospital of Shijiazhuang City, Shijiazhuang, China

9. Jianshu Feng,

The Third Hospital of Shijiazhuang City, Shijiazhuang, China

10. Feng Li,

The Third Hospital of Shijiazhuang City, Shijiazhuang, China, Email:690153442@qq.com 